\title{
Using an Ontology to Suggest Software Design Patterns Integration
}

\author{
Dania Harb, Cédric Bouhours, Hervé Leblanc \\ IRIT - MACAO \\ Université Paul Sabatier \\ 118 Route de Narbonne \\ F-31062 TOULOUSE CEDEX 9 \\ \{harb, bouhours, leblanc\}@irit.fr
}

\begin{abstract}
To give a consistent and more valuable feature on models, we propose that model-driven processes should be able to reuse the expert knowledge generally expressed in terms of patterns. In order to formalize and use them, some design pattern ontologies have been developed. To share them on the Web they have been implemented using the OWL language. They can be easily interrogated with dedicated query languages. Our work has consisted in extending a design pattern intent ontology with "alternative model" and "strong points" concepts, which partially refers "anti-patterns". We validate this approach in tooling a step of a design review activity, we have proposed. This activity, directed by design patterns, is adapted to a model driven process, for the need to improve object-oriented architecture quality.

Keywords: OWL, SPARQL, Software Design Pattern, Design Review Activity, MDE, MDA
\end{abstract}

\section{Introduction}

The emergent MDE community, aiming at giving a productive feature on models, has proposed model-driven process development. However, to obtain guarantees on model relevance at the end of each activity, these processes should promote the reuse of the knowledge of experts generally expressed in terms of analysis [1], design [2] or architectural [3] patterns approved by the community. Given the existence of "code review" activities [4] in some development processes, we have specified a "design review" activity [5] directed by design patterns and oriented to model quality. In this activity, we propose to parse models to find fragments substitutable with software design patterns and to replace them if the intent of the designer matches with the intent of the pattern and if the architectural qualities of the pattern are needed. Our activity is situated after the design stage, and its purpose is to urge and to help the designer to integrate design pattern in his design.

Thanks to their Design Pattern Intent Ontology (DPIO), Kampffmeyer et al. [6] have developed a wizard enabling designers to efficiently retrieve software design patterns applicable for their design problems, during the design stage. Our approach 
has not the same timing. It is situated after the design stage, and it verifies if there is no known bad design practices in a model. So, the designer is not in need of identifying design problems, it is the activity which finds the lacks in his models and suggests design patterns integrations instead. However, the DPIO [6] is an interesting start point for the formalization of our concepts because it links software design pattern to some problem concepts. So, in reusing this ontology backwards (from the pattern to the design problems), and in adding our concepts, we are able to establish a dialog with the designer.

In this paper, after presenting the design review activity, we explain how we have reused and extended the DPIO [6]. We illustrate the execution of our activity on a "file system management" example.

\section{The Design Review Activity}

The design review activity, presented in [5], may be decomposed into four steps (see Fig. 1)

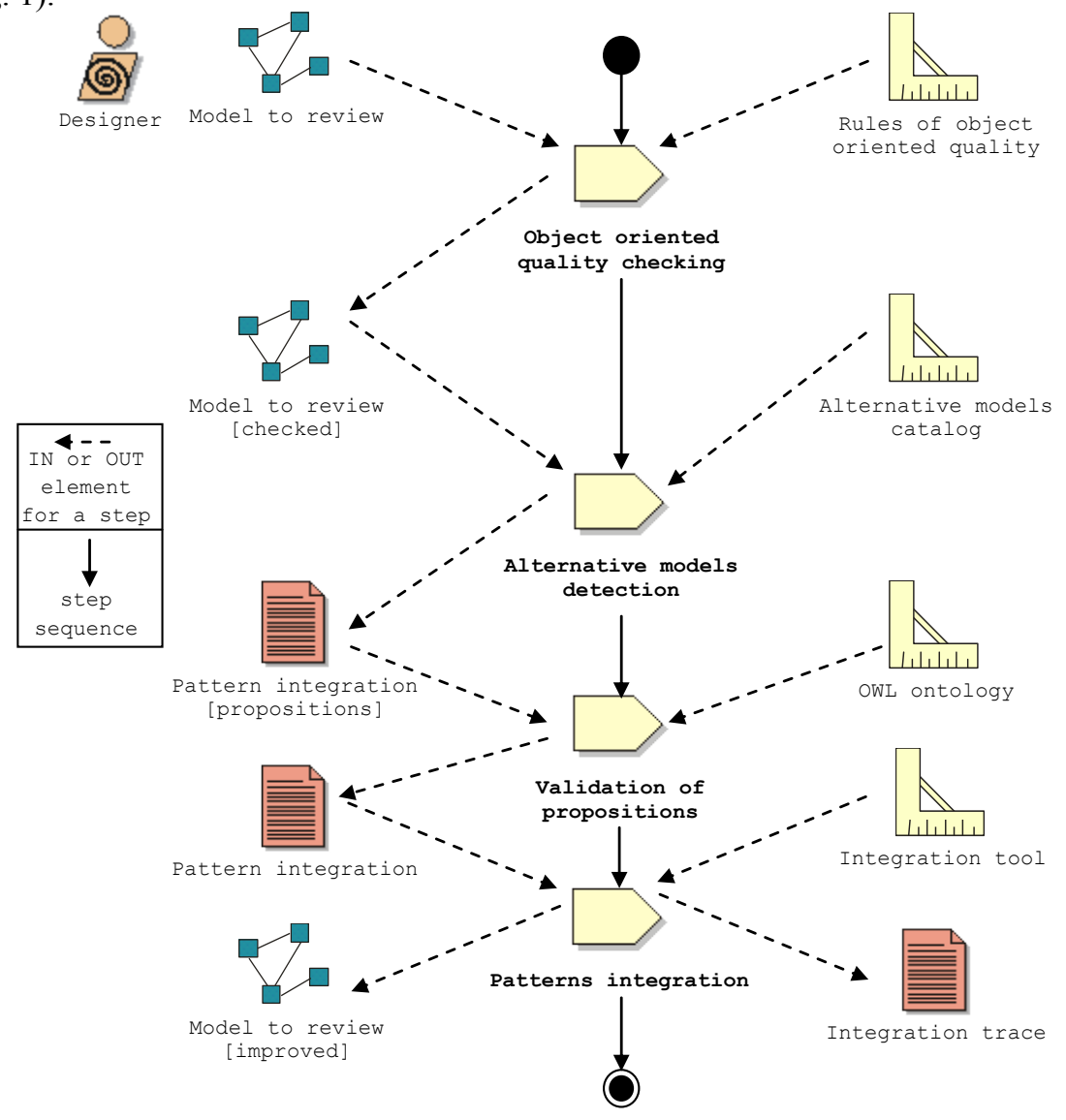

Fig. 1. Design Review Activity 
In order to work with models in a "sufficient" quality, the first step checks good basic object-oriented design practices.

When the model to review is checked in a "sufficient" quality state, the second step consists in an automatic research of model fragments which are candidate to a substitution with a pattern. This research is based on structural similarities detection with "alternative models". An "alternative model" is a model which solves inadequately the same problem as a pattern [5]. That means there is a better solution to solve this problem. Our work hypothesis is that a software design pattern is the best solution for a given design problem. According to the taxonomy proposed by Chikofsky and Cross [8], our detection technique can be connected to a redocumentation technique as to permit model restructuring. Our "alternative models" catalog is presented in [9], with the experiments used to constitute it.

Each "alternative model" detected in the model represents propositions of fragments substitutable with a design pattern. Since we need the designer opinion in the third step, our ontology will help him determine if his intent matches with the suggested pattern intent and whether the propositions are needed in the model to review.

With the designer authorization, the last step consists in integrating the validated propositions into the model. This integration is done thanks to an automatic model refactoring.

\section{Reusing and extending an existing ontology}

In order to improve the design of object oriented models, our work relies on detecting all instances of alternative models in UML design models and substituting them, if necessary, with appropriate design patterns. Each class of the instances detected is connected in the same manner as the classes of the alternative model. So, since the detection is only structural, the instances detected must be checked by the designer before any substitution with a pattern. Therefore, after the detection step, propositions of patterns integration consist of sets of model fragments representing a possible substitution. These sets may be large where some fragments may not be relevant with a substitution. So, to help the designer in filtering the fragments, we need an ontology that formalizes intent of design patterns (is the substitution have a sense?) and our characterizations of "alternative models" in terms of quality features (is the effort of the substitution balanced by improved architectural qualities?).

For this purpose, we choose OWL, the Web Ontology Language [10], to import an existing ontology on design patterns intent and extend it by adding our knowledge on "alternative models". We validated our new knowledge base using a specific query language to interrogate it and filter out the pertinent information. 


\subsection{Requirements}

Our catalogue is composed with "alternative models", introduced in Section 2, and their characterization. We have constituted our catalog in asking students to solve some design problems. These problems were simply solvable with designs patterns, but, as the students chosen have no knowledge on design patterns, they solve the problems without using design patterns. In following our work hypothesis, their solutions were not the best solution for the problem, and so, the models produced had some design defects. The characterization of these defects consists in a valuation of the "strong points" of the pattern. "Strong points" are criteria of object-oriented architecture or software engineering quality, partially deduced from the "consequences" section of the GoF [2] catalogue and from our study on the design defects of "alternative models". As pattern injection may alter some object-oriented metrics [11], "strong points" allow us to compute dedicated pattern metrics to classify the "alternative models" and to help the estimation of the pertinence of pattern injection in a design model. Each "alternative model" perturbs the "strong points" of its associated pattern.

Since we need to formally describe design patterns, "alternative models" and "strong points" in a machine readable format, we start with the DPIO ontology. These concepts must be constructed in a way that allows querying based on the "alternative model" detected.

Intent: Compose objects into tree structures to represent part-whole hierarchies. Composite lets clients treat individual objects and compositions of objects uniformly.

Applicability: Use the Composite pattern when:

- you want to represent part-whole hierarchies of objects.

- you want clients to be able to ignore the difference between compositions of objects and individual objects. Clients will treat all objects in the composite structure uniformly.

\section{Structure:}

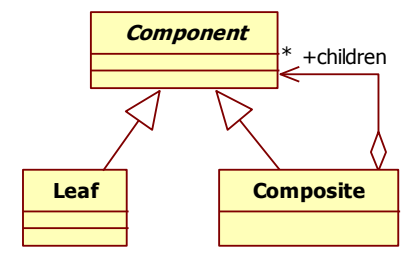

\section{Strong points:}

1 Decoupling and extensibility

1.1 Maximal factorization of the composition

1.2 Addition or removal of a Leaf does not need code modification

1.3 Addition or removal of a Composite does not need code modification

2 Uniform processing

2.1 Uniform processing on operations of composed object

2.2 Uniform processing on composition managing

2.3 Unique access point for the client 
Fig. 2. Composite Pattern and its "Strong Points"

In Fig. 2, we present one of the GoF patterns, named Composite. The intent, the applicability and the structure are provided directly from the GoF book while the "strong points" are deduced from our experiments by comparing solutions to specific design problem implemented by the Composite pattern and its "alternative models". Fig. 3 shows the structure and the design defect valuation of an "alternative model" to the Composite pattern. We have named it "Development of the composition on Composite with process conformance" in reference of its design defects. Then an "alternative model" can be considered as a "chipped pattern".

So we have made two major hypotheses about "alternative models". First, each "alternative model" is attached by the valuation of their design defects to a unique design pattern. Second, each "alternative model" has one or more strong points perturbed. We assume that the same structure of an "alternative model" can be duplicated in our catalog, but with a different name, a different valuation and some different components.

\section{Name:}

Development of the composition on Composite with process conformance

\section{Alternative model:}

\begin{tabular}{|l|l|l|l|}
\hline \multicolumn{4}{|c|}{ Strong points perturbations } \\
\hline 1.1 & $\varkappa$ & 2.1 & $\vee$ \\
\hline 1.2 & $\varkappa$ & 2.2 & $\vee$ \\
\hline 1.3 & $\varkappa$ & 2.3 & $\vee$ \\
\hline
\end{tabular}

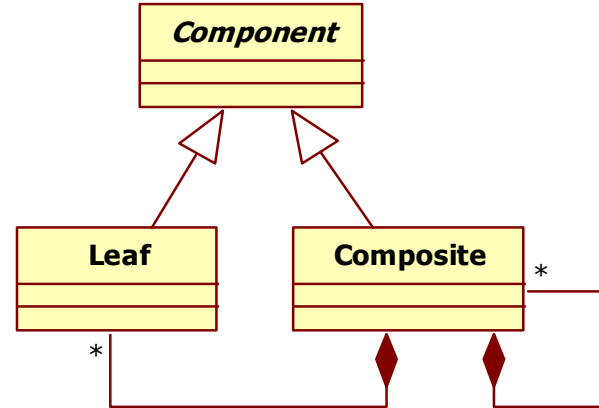

Fig. 3. Characterization of an "Alternative Model"

\subsection{Existing ontology: the Design Pattern Intent Ontology}

Design patterns have been used successfully in recent years in the software engineering community in order to share knowledge about the structural and behavioural properties of software. Most of the existing approaches to formalizing design patterns are based on structural aspects. For example, the work of Dietrich et al. [12] uses the OWL to formally describe the structure of design patterns and then transform it in first-order logic predicates which are reuse as an entry for a scanner pattern. However, there is more lightly approaches concentrated in the usability of design patterns according to the design problems they solve. Kampffmeyer and Zschaler [6] define the intent of the 23 GoF design patterns [2] using OWL. Their 
work was based on the work of Tichy [13], who developed a catalogue of more than hundred design patterns classified according to the problems patterns solve.

The core structure of the DPIO, provided from the paper [6], is presented in Fig. 4 by UML classes and associations. Kampffmeyer and Zschaler chose to represent their ontology with UML diagram because they consider that is easily to understand. To read the diagram, they indicate: "The relations between DesignPattern, DPProblem and ProblemConcept classes are depicted using UML-notations. UML classes symbolize OWL classes and UML associations symbolize OWL object properties. Each DesignPattern is a solution to one or more design pattern problem DPProblem. The association between them indicates an object property isSolutionTo which is an inverse property of isSolvedBy. DPProblem is defined that is the root node for more specific problems. The association class Constrains indicates an OWL object property that can be specialized also by subproperties. DPProblem is a set of classes that describe a problem by constraining a ProblemConcept". The DPIO contains the vocabulary for describing the intent of design patterns.

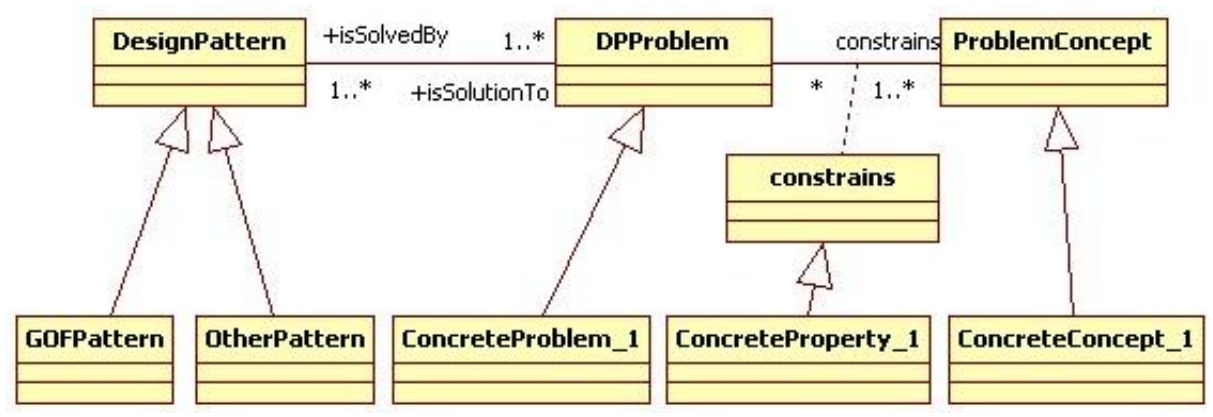

Fig. 4. Graphical overview of the core structure of the DPIO

All the 23 GoF patterns inherit from the DesignPattern class. DPProblem and ProblemConcept are the root classes of the other hierarchies.

Based on the work of [6], and instead of finding the design pattern for a given problem, we retrieve the intent of a design pattern. It is much like reversing the query to get the pertinent data from the same ontology. So we can benefit from their existing work and their published ontology.

\subsection{Method and Results}

Now to determine the scope of our new ontology, there are kinds of questions called "competency questions" the ontology should be able to answer [14]. Our work could be defined in 3 steps: first, when an "alternative model" is detected, we need to interrogate our knowledge base to know which design pattern could replace it. Second, we will verify with the designer if his "alternative model" detected has a similar intent as the corresponding design pattern. Last, in this case, we will show him 
the lack in his model by displaying the perturbed "strong points". Then, if the designer finds the need to improve his model, his fragment will be substituted with the design pattern. Therefore, the three competency questions are as follow:

1. Which design pattern could replace a given "alternative model"?

2. What is the intent of the corresponding design pattern?

3. Which are the "strong points" perturbed using this "alternative model"?

In designing the structure of the new ontology, we took into consideration all the possible relations between the classes in the DPIO model and the classes we want to add:

1. Each"alternative model" could be replaced by one and only one Design Pattern. But a Design Pattern will replace one to many "alternative models".

2. An "alternative model" perturbs at least one "strong point" of the Design Pattern that can replace it.

From this analysis, we extend the DPIO by adding our new concepts.

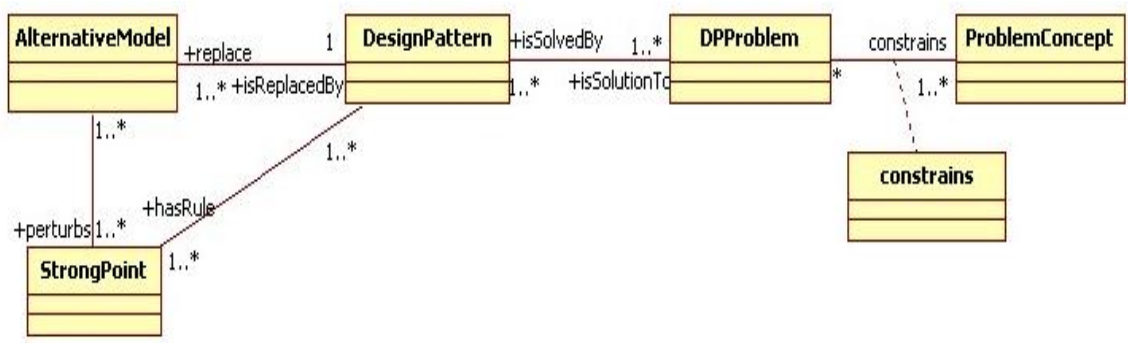

Fig. 5. Graphical overview of the structure of the extended ontology

Fig. 5 represents the new structure of the extended ontology. Based on this structure and the relations between classes, we extended the existing ontology with OWL classes and properties as follow:

1. Two new OWL classes:

a. AlternativeModel: the root class of all "alternative models". They are grouped by the design pattern that could replace them. For example, we find six "alternative models" for the Composite pattern. They inherit all from the Composite_AM class (Fig. 6). They have the name of their super class followed by their numeration in the catalogue.

b. StrongPoint: the root class of all the "strong points". They are attached to a design pattern. For example, we find two main "strong points" for the Composite pattern: Composite Rule 1 and Composite_Rule_2 (Fig. 6); each one of them was précised by three sub features. They have the name of their super class followed by their numeration in the catalogue. 
2. Four new OWL properties:

a. isReplacedBy: links an AlternativeModel to his corresponding DesignPattern.

b. Replace: the inverse of isReplacedBy.

c. Perturbes: links an AlternativeModel to the valuation of the corresponding pattern "strong points" (StrongPoint).

d. hasRule: links a DesignPattern class to one of its StrongPoint.

Fig. 6 shows a detailed structure of the extended base concerning the Composite pattern. The "alternative model" presented in Fig. 3 perturbs the three subfeatures of the first "strong point" of the Composite pattern that concerned in the Decoupling and extensibility. More precisely, for each OWL class concerning our concepts, we have:

\begin{tabular}{|l|l|}
\hline OWL Classes & $\boldsymbol{r d f s : c o m m e n t}$ \\
\hline Composite_AM_5 & $\begin{array}{l}\text { Development of the composition on "Composite" with } \\
\text { protocol conformance }\end{array}$ \\
\hline Composite_Rule_1 & Decoupling and Extensibility \\
\hline Composite_Rule_2 & Uniform processing \\
\hline Composite_Rule_1.1 & Maximal factorization of the composition \\
\hline Composite_Rul__1.2 & $\begin{array}{l}\text { Adding or removing a Leaf does not need a code } \\
\text { modification }\end{array}$ \\
\hline Composite_Rule_1.3 & $\begin{array}{l}\text { Adding or removing a Composite does not need a code } \\
\text { modification }\end{array}$ \\
\hline Composite_Rule_2.1 & Uniform processing on operations of composed objects \\
\hline Composite_Rule_2.2 & Uniform processing on compositions management \\
\hline Composite_Rule_2.3 & Unique access point for the client \\
\hline
\end{tabular}

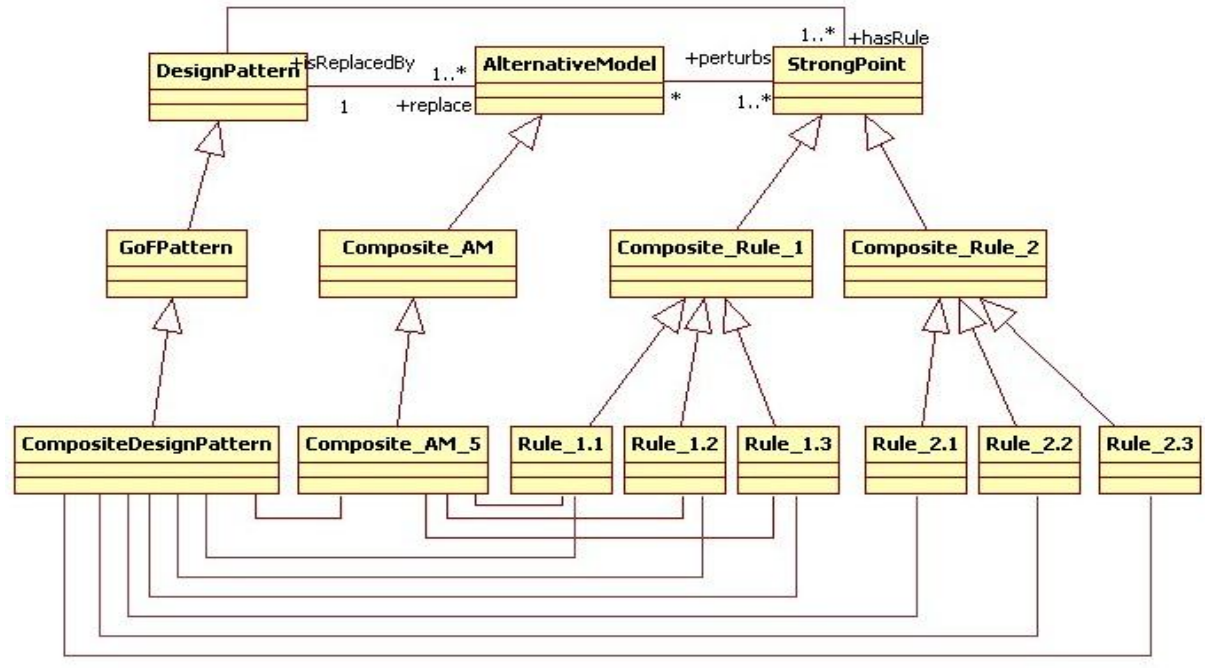

Fig. 6. Detailed structure of the extended ontology 
For presentation reasons, we have omitted the name of the design pattern in each sub feature.

We used Protégé [15], an open source ontology editor and knowledge-base framework, to load the existing ontology and add our new classes, properties, property characteristics, and interrogate it using queries. We referred to a user guide [14] on how to develop an ontology using Protégé and the OWL Plug-in. We created our OWL classes, linked them by OWL properties, and interrogated the knowledge base by generating SPARQL (SPARQL Protocol and RDF Query Language) [16] queries to answer our competency questions.

$S P A R Q L$ is a W3C Candidate Recommendation towards a standard query language for the Semantic Web. Its focus is on querying RDF graphs at the triple level. $S P A R Q L$ can be used to query an RDF Schema or OWL model to filter out individuals with specific characteristics.

\section{Illustration on a "File System Management" Design}

After adding our new concepts to the DPIO, the knowledge base could now be interrogated according to the competency questions we mentioned earlier. Standard ontology reasoning is used to retrieve the results responding to queries. In order to illustrate the use of the ontology, we execute the whole activity on an example. It was found in a subject of an object-oriented programming supervised practical work. It aims to implement a file management system represented in the Fig. 7 below.

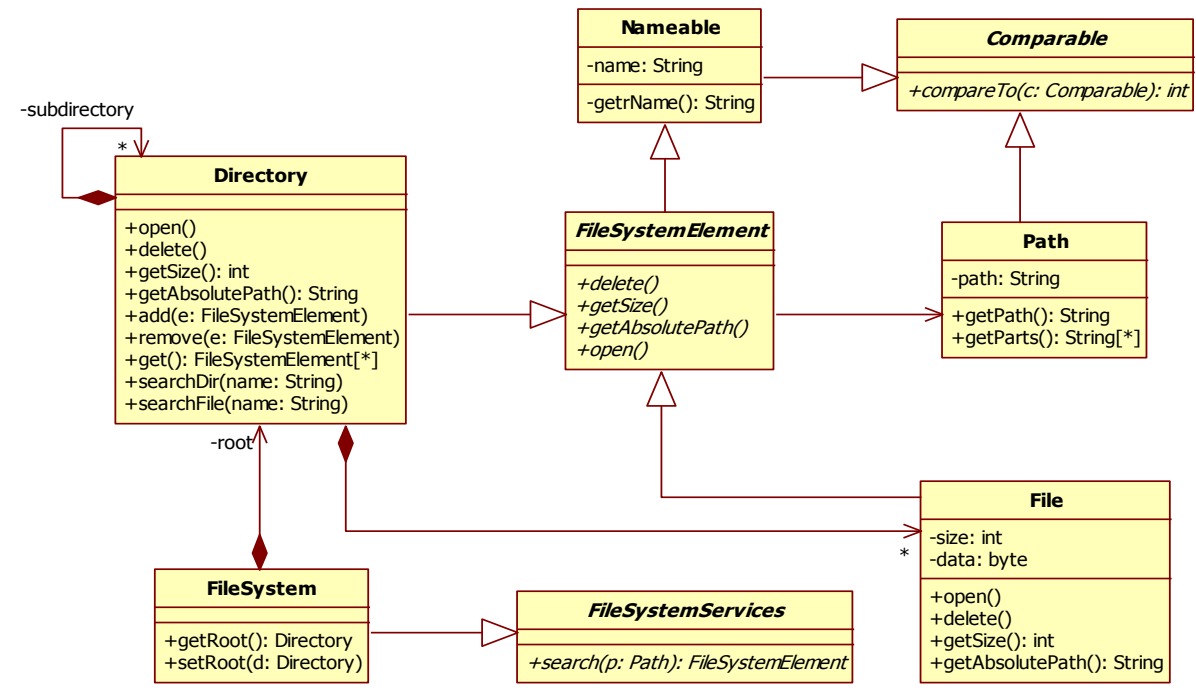

Fig. 7. Model to Review: File System Management 
This static UML model represents a basic architecture for a File System Management. Authors of this model are interested in the presentation of some object concepts:

- Inheritance between classes and abstract classes. A uniform protocol for every FileSystemElement is encapsulated by a corresponding abstract class. Directories and Files must respect this protocol via inheritance relationship. We can note that all concrete classes are derived directly or indirectly from an abstract class. This rule enforces the emergence of reusable protocols.

- Management of references, here composition links, between container and components. A Directory object manages some references to Files and Directories objects.

Nevertheless, this model contains a misconception. Although there is a uniform protocol owned by the class FileSystemElement, the management of composite links along a hierarchical structure is duplicated. Indeed, Directory class manages independently links on Files and Directories. Now, we consider two evolution scenarios. The first is adding new Terminal types in the tree structure, for example, symbolic links in UNIX systems. This evolution requires the management of this new type of links by the Directory class and then requires code modification and code duplication in this class. The second is adding new non Terminal types in the tree structure, for example archive files in UNIX or in Java environment. We can consider that an archive file has the same functionalities as a Directory. This evolution requires a reflexive link on an archive file class and the duplication of all links that represent composition links in the tree structure. Then it requires duplication of management of composition and modification in the Directory class, it must manage another type on FileSystemElement. These two scenarios show a decoupling problem (each container manages a part of the composite structure) and an extensibility limitation (it requires existing code modification for adding new type of terminal or non terminal element of the composition hierarchy). Therefore, this model can be improved. Furthermore, when the authors have implemented this model, they realized that there were defects, and they adapted their code to improve it.

\subsection{Object-Oriented Quality Checking}

Visually, there is no design mistake: each class of the model presents a reusable protocol. Composition links are used here as delegation between Directory and File. And messages sent between them have the same selector.

\section{2 “Alternative Models" Detection}

This step consists in the execution of all queries corresponding at each "alternative model" of the base. In this example, the query of the fifth Composite "alternative model" returns theses matching classes:

1. The Directory class is able to play the role of the Composite class.

2. The File class is able to play the role of the Leaf Class.

3. The FileSystemElement is able to play the role of the Component class. 
This means that we detected an "alternative model" for the Composite pattern because they have the same structural features (cf. Fig. 8).
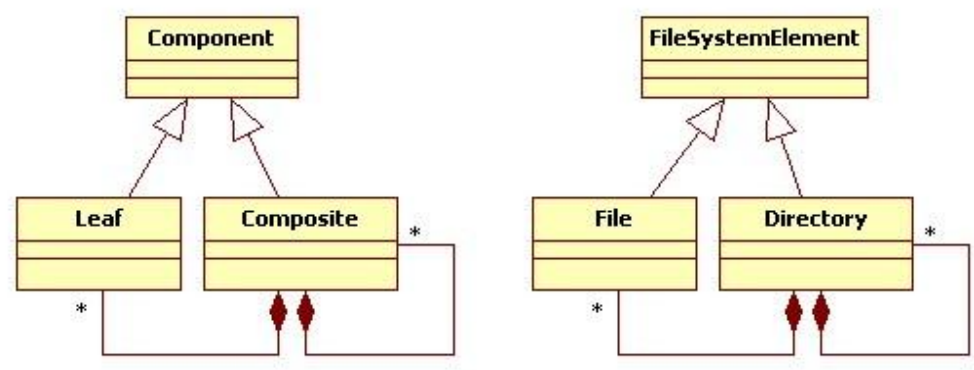

Fig. 8. The fifth Composite "Alternative Model" its Instantiation in the Model

\subsection{Designer/Machine dialog}

At this step, the designer must verify the substitutability of the detected fragment. Firstly, he must verify if the intent of the fragment matches with the proposed design pattern. To do so, we build a question thanks to a SPARQL query we have coded (cf. Listing 1). This query retrieves the intent of the design pattern in using the "alternative model" detected (here Composite_AM_5). Indeed, we consider that the intent of the pattern is described with a list of couples (constraint - ProblemConcept) in the ontology (see Fig. 5).

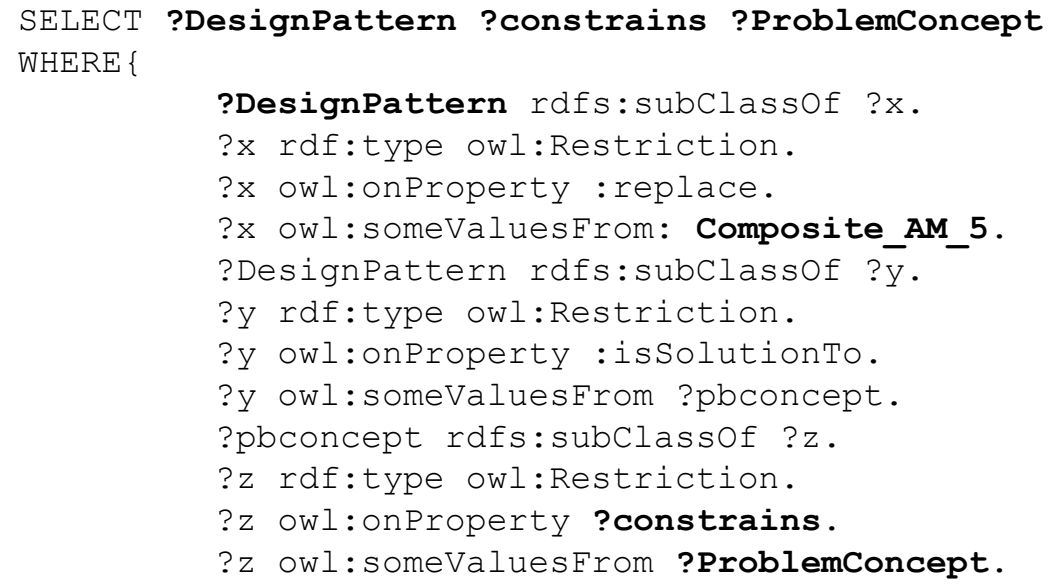

Listing 1 SPARQL query to retrieve the intent of the Composite pattern that could replace the "alternative model" Composite_AM_5 
Based on the results (cf. Fig. 9) of this query, we will proceed in dialoguing the designer with the first question: We have detected in your design an alternative model of the CompositeDesignPattern. Is the fragment \{FileSystemElement, File, Directory\} composes Object, builds TreeStructure and nests Objects?

\begin{tabular}{|l|l|l|}
\hline \multicolumn{1}{|c|}{ Results } & \multicolumn{1}{c|}{ ProblemConcept } \\
\hline \multicolumn{1}{|c|}{ DesignPattern } & \multicolumn{1}{|c|}{ constrains } & \multicolumn{1}{c|}{ Pbject } \\
\hline CompositeDesignPattern & $\square$ composes & TreeStructure \\
CompositeDesignPattern & $\square$ builds & Object \\
CompositeDesignPattern & $\square$ nests & Obls \\
\hline
\end{tabular}

Fig. 9. Screenshot of Protégé after executing the query (Listing 1)

We can note that the intent of \{FileSystemElement, File, Directory\} is a recursive composition: "Directories are composed with Files or Directories which are composed with...". So the answer to the previous question is positive.

Now, we must check the interest to replace the fragment with the pattern. Thanks to the perturbation of the "strong points", we can present to the designer the advantage to use the pattern. We retrieve the perturbed "strong points" with a SPARQL query (Listing 2):

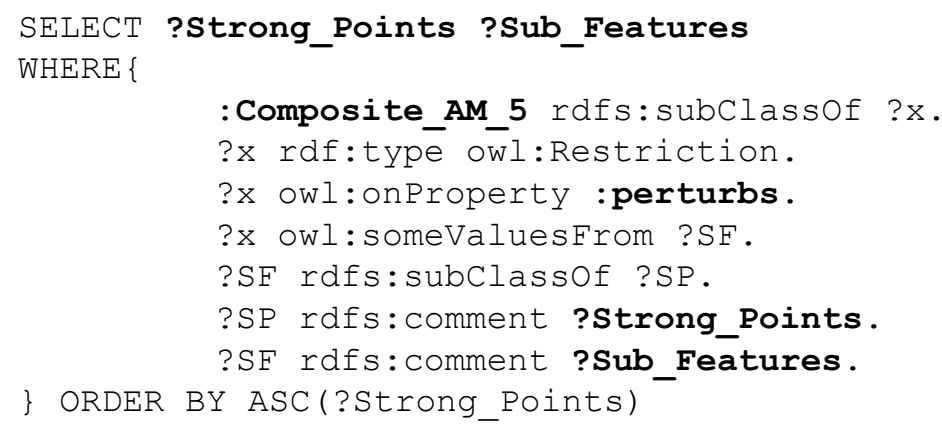

Listing 2 SPARQL query to retrieve the "strong points" perturbed by COMPOSITE_AM_5

The second question is built with the results (cf. Fig. 10) of the previous query: Our analysis shows that you have problems of "Decoupling and Extensibility"; your model is unable to satisfy those points:

1. Maximal factorization of the composition.

2. Addition or removal of a leaf does not need code modification.

3. Addition or removal of a composite does not need code modification.

In injecting the CompositeDesignPattern, you will improve all of these points. Do you want to refactor the identified fragment \{FileSystemElement, File, Directory\}? 


Results
\begin{tabular}{|l|l|}
\hline \multicolumn{1}{|c|}{ Strong_Points } & \multicolumn{1}{c|}{ Sub_Features } \\
\hline $\begin{array}{l}\text { Decoupling and Extensibility } \\
\text { Decoupling and Extensibility } \\
\text { Decoupling and Extensibility }\end{array}$ & Maximal factorization of the composition \\
\hline & Addition or removal of a Leaf does not need code modification \\
\hline
\end{tabular}

Fig. 10. Screenshot of the result window presenting the "strong points" perturbed

As we consider that the model may evolve, it is useful to guarantee that there are extensibility and decoupling possibilities. Therefore, the fragment must be substituted with the pattern.

\subsection{Patterns Integration}

At this step, the identified fragment is replaced by the suggested design pattern like the Fig. 11 below:

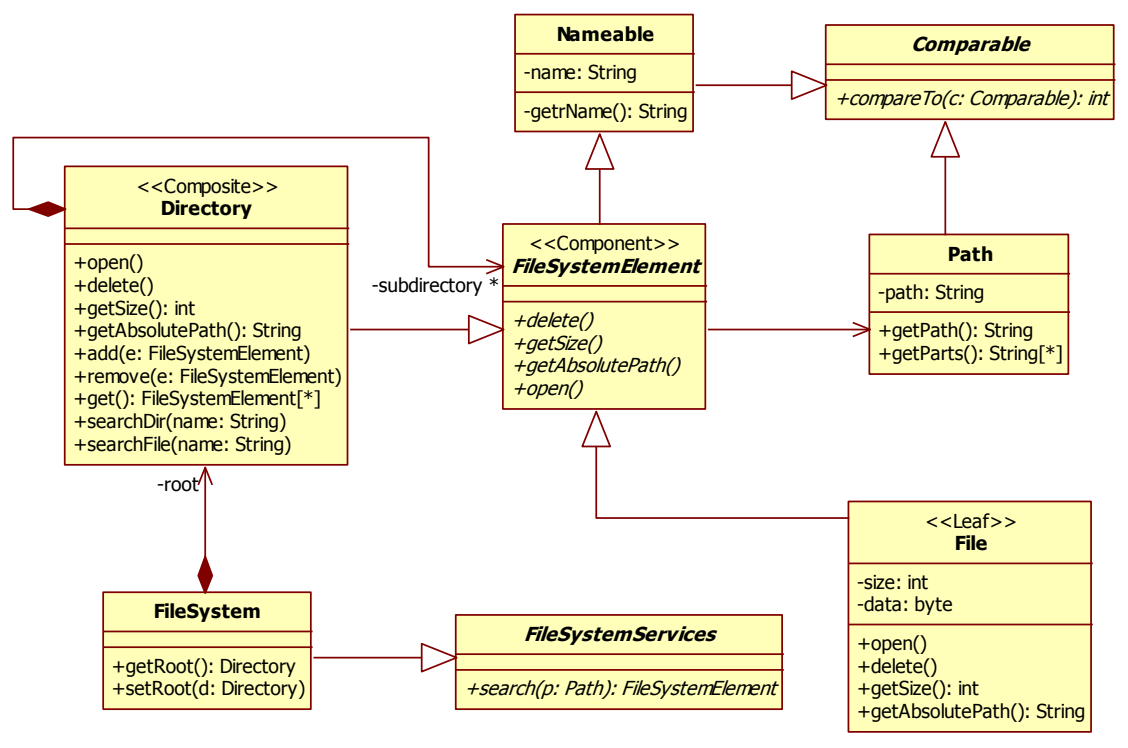

Fig. 11. Model to Review Improved

To do so, a suite of simple model refactoring suffices to integrate the pattern. Here, it consists in:

- Remove composition link between Directory and File.

- Move the end of the recursive composition link from Directory to FileSystemElement.

These inter-classes refactorings can be automatically deduced with an operation of "differentiation" between the "alternative model" and the pattern structure. 
At the end of the activity, we can say that this model is improved, because we have substituted a fragment (with "weak points") with a pattern (with "strong points"). This transformation may appear as non fundamental in the model, but at the code level, the implications are substantial. Every hierarchy traversal methods are simpler to implement, and there is less code to write. Moreover, in case of extensions, there is no code modification of existing classes.

\section{Conclusion and Perspectives}

The approach of reusing and extending an existing ontology corresponding to our requirements was successfully applied. From the existing DPIO ontology, we have plugged our concepts on "alternative models" and "strong points". These concepts are fundamental for tooling our Design Review Activity. Accurately, at the step named validation of substitution propositions, we have simulated a dialog with a designer by interrogating the extended base using queries. These queries will be generated automatically by a template process. The integration of this work into a tool dedicated to the design review activity is envisaged.

Finally, we conclude with some perspectives:

- Take into consideration the relationships between patterns. For example, the Decorator pattern can be applied to the Composite pattern structure.

- Take into consideration the applicability of each pattern. For example, referring to the GoF book, one of the applicability of the Composite pattern is: you want clients to be able to ignore the difference between compositions of objects and individual objects. We notice that this sentence cannot be part of the pattern intention but can be considered as a "strong point".

- Optimize our knowledge base by sharing common "strong points" between patterns. For example, the Composite, the Decorator and the Bridge pattern have a same "strong point" concerning the maximal factorization between specific classes.

- Use inference rules to find new concepts when adding new "alternative models" or "strong points". This could help us improving our knowledge on patterns and particularly, our knowledge on the good practices on object oriented architecture.

\section{Acknowledgements}

We are grateful to Mrs. Nathalie Aussenac-Gilles for her precious advices during this work. 


\section{References}

1. Fowler M., "Analysis patterns: reusable objects models", Addison Wesley Longman Publishing Co, Inc., 1997.

2. Gamma E., Helm R., Johnson R., Vlissides J., "Design Patterns: Elements of Reusable Object-Oriented Software”, Addison Wesley Professional, 1995.

3. Buschmann F., Meunier R., Rohnert H., Sommerlad P., Stal M., "Pattern-Oriented Software Architecture", John Wiley \& Sons, August 1996.

4. Dunsmore A.P., "Comprehension and Visualisation of Object-Oriented code for Inspections", Technical Report, EFoCS-33-98, Computer Science Department, University of Strathclyde, 1998.

5. Bouhours C., Leblanc H.., Percebois C., "Alternative Models for a Design Review Activity". In : Workshop on Quality in Modeling - ACM/IEEE International Conference on Model Driven Engineering Languages and Systems, NASHVILLE, TN (USA), 30/09/2007-05/10/2007, Ludwig KUZNIARZ, Jean-Louis SOURROUILLE, Miroslaw STARON (Eds.), Springer, p. 65-79, October 2007.

6. Kampffmeyer H., Zschaler S., Engels G., Opdyke B., Schmidt D. C., Weil F., "Finding the Pattern You Need: The Design Pattern Intent Ontology", in MoDELS, Springer, 2007, volume 4735, pages 211-225.

7. Guéhéneuc Y. G., Albin-Amiot. H., "Using Design Patterns and Constraints to Automate the Detection and Correction of Inter-Class Design Defects", in Proceedings conference TOOLS, July 2001, pages 296-305.

8. Chikofsky E. J., Cross J. H., "Reverse engineering and design recovery: A taxonomy", in IEEE Software, 7(1), page 13-17, January 1990.

9. Bouhours C., Leblanc H., Percebois C., "Alternative Models for Structural Design Patterns", research report, IRIT/RR--2007-1--FR, IRIT, December 2007, http:/www.irit.fr/recherches/DCL/MACAO/docs/AlternativeModelsForStructuralDesignPa tterns.pdf.

10. D.L. McGuinness and F. van Harmelen: OWL Web Ontology Language Overview, 2004.http://www.w3c.org/TR/owl-features/

11. Huston B., "The effects of design pattern application on metric scores", in Journal of Systems and Software, 58(3), Elsevier Science, September 15, 2001, pages 261-269.

12. Dietrich, J., Elgar, C.: A formal description of design patterns using OWL, in: Australian Software Engineering Conference (ASWEC'05), pp. 243-250. IEEE Computer Society, Los Alamitos, 2005. http://doi.ieeecomputersociety.org/10.1109/ASWEC.2005.6

13. Tichy, W.F.: A catalogue of general-purpose software design patterns. In: TOOLS'97. Proceedings of the Tools-23: Technology of Object-Oriented Languages and Systems, IEEE Computer Society, Washington, DC, USA, 1997.

14. Noy, N.F., McGuinness, D.L.: Ontology development 101: A guide to creating your first ontology. Technical Report KSL-01-05, Knowledge Systems Laboratory, Stanford University, Stanford, CA, 94305, USA, March 2001.

15. Protégé ontology editor and knowledge acquisition system (2006). $\mathrm{http}: / /$ protege.stanford.edu/

16. Prud'hommeaux E., Seaborne: SPARQL Query Language for RDF, January 2008. http://www.w3.org/TR/rdf-sparql-query/ 